\title{
Efficient attenuation of beam vibrations by inertial amplification
}

\author{
Barys, Mateusz; Jensen, Jakob S.; Frandsen, Niels Morten Marslev
}

Published in:

European Journal of Mechanics A - Solids

Link to article, DOI:

10.1016/j.euromechsol.2018.04.001

Publication date:

2018

Document Version

Peer reviewed version

Link back to DTU Orbit

Citation (APA):

Barys, M., Jensen, J. S., \& Frandsen, N. M. M. (2018). Efficient attenuation of beam vibrations by inertial amplification. European Journal of Mechanics A - Solids, 71, 245-257.

https://doi.org/10.1016/j.euromechsol.2018.04.001

\section{General rights}

Copyright and moral rights for the publications made accessible in the public portal are retained by the authors and/or other copyright owners and it is a condition of accessing publications that users recognise and abide by the legal requirements associated with these rights.

- Users may download and print one copy of any publication from the public portal for the purpose of private study or research.

- You may not further distribute the material or use it for any profit-making activity or commercial gain

- You may freely distribute the URL identifying the publication in the public portal

If you believe that this document breaches copyright please contact us providing details, and we will remove access to the work immediately and investigate your claim. 


\section{Accepted Manuscript}

Efficient attenuation of beam vibrations by inertial amplification

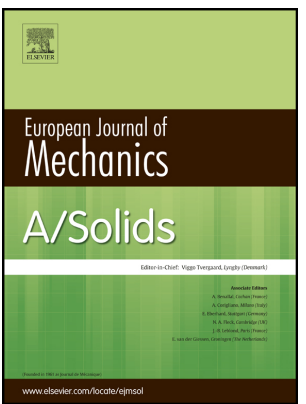

Mateusz Barys, Jakob S. Jensen, Niels M.M. Frandsen

PII: S0997-7538(17)30696-4

DOI: $\quad$ 10.1016/j.euromechsol.2018.04.001

Reference: EJMSOL 3581

To appear in: European Journal of Mechanics / A Solids

Received Date: 25 September 2017

Revised Date: 29 March 2018

Accepted Date: 2 April 2018

Please cite this article as: Barys, M., Jensen, J.S., Frandsen, N.M.M., Efficient attenuation of beam vibrations by inertial amplification, European Journal of Mechanics / A Solids (2018), doi: 10.1016/ j.euromechsol.2018.04.001.

This is a PDF file of an unedited manuscript that has been accepted for publication. As a service to our customers we are providing this early version of the manuscript. The manuscript will undergo copyediting, typesetting, and review of the resulting proof before it is published in its final form. Please note that during the production process errors may be discovered which could affect the content, and all legal disclaimers that apply to the journal pertain. 


\title{
Efficient attenuation of beam vibrations by inertial amplification ${ }^{\text {th }}$
}

\author{
Mateusz Barys ${ }^{\mathrm{a}, *}$, Jakob S. Jensen ${ }^{\mathrm{b}}$, Niels M.M. Frandsen ${ }^{\mathrm{c}}$ \\ ${ }^{a}$ Department of Automotive and Construction Machinery Engineering, Institute of Machine Design Fundamentals, Warsaw University of Technology, Warsaw, \\ Poland \\ ${ }^{b}$ Department of Electrical Engineering, Centre for Acoustic-Mechanical Micro Systems, Technical University of Denmark, 2800 Kgs. Lyngby, Denmark \\ ${ }^{c}$ Department of Mechanical Engineering, Section for Solid Mechanics, Technical University of Denmark, 2800 Kgs. Lyngby, Denmark
}

\begin{abstract}
We demonstrate efficient attenuation of flexural vibrations by attaching a simple inertial amplification (IA) mechanism to a slender elastic beam. The mechanism generates enhanced inertial forces between two attachment points, which effectively counteracts the elastic forces in the beam for certain anti-resonance frequencies. These anti-resonances may be generated in the low-frequency range, even for a small added mass. Furthermore, the hybrid structures are shown to exhibit two neighbouring anti-resonance dips providing wide and deep attenuation regions in the frequency domain. The obtained numerical results are validated with the experimental data.
\end{abstract}

Keywords: Inertial amplification mechanism, Bending vibrations, Vibration attenuation

\section{Introduction}

The use of locally resonating structures for vibration mitigation has received great attention in literature within the last one hundred years. The idea is based on the transfer of vibrational energy to a resonator that vibrates with a frequency tuned to a particular critical frequency of the main structure. The first attempts to apply the concept, in the form of the so-called tuned mass dampers (TMD), date back to 1909 in the work by Frahm [1]. He applied the TMD's to a ship in order to reduce its rolling motion and hull vibrations. However, the first theoretical treatment of the TMD was presented nearly 10 years later in 1928 by Den Hartog and Ormondroyd [2]. In 1940, Den Hartog's book [3] was published, in which optimal tuning parameters were derived and since then TMD's, and dynamic vibration absorbers in a more general framework, have been comprehensively studied by e.g. Randall [4], Warburton [5], Tsai and Lin [6], Connor and more [7].

A main challenge when designing dynamic vibration absorbers is to obtain a wide region of vibration attenuation in the low-frequency range. In principle, anti-resonances, and hence vibration attenuation, can be created using a local resonance at an arbitrary low frequency. However, in practice the lower limit is bounded by spatial limitations and the width of the frequency range affected by the resonator is severely restricted.

In 1967, Flannelly patented a device for attenuating vibrations called a "Dynamic Antiresonant Vibration Isolator" (DAVI) [8]. The device is similar to a single degree of freedom (sdof) mass-spring system, however rather than utilizing springs, the mass is embedded between the main mass and the

\footnotetext{
This document is a collaborative effort

${ }^{*}$ Corresponding author

Email address: mateuszbarys6@gmail.com (Mateusz Barys)
}

ground via a lever mechanism. The inertial force from the isolation mass is amplified by the lever, and the system works as a mechanical low-pass filter, effectively isolating the main mass from ground vibrations above a particular filter frequency. Unlike the usual spring-mass system, where the filter frequency is governed by the stiffness and mass of the attached device, the performance of the DAVI system is governed by the isolation mass and lever ratio, both of which increases the inertial forces generated by the device. The lack of an added spring means that no additional resonances are created. This also implies that the "effective" mass may be fairly large without having to use an impractically large physical mass. In this way anti-resonance frequencies in the low-frequency range are more feasible.

Based on an electrical-mechanical analogy, Smith developed the "inerter" in 2002 [9], which is the inertial equivalent of the spring and dashpot. Work on the new device was apparently motivated by an electrical analogy, but the working principle is similar to Flannelly's system. The inerter provides a force proportional to the relative acceleration between two attachment points for vibration isolation purposes. The invention has been used in the suspension systems of Formula One cars, showing practical applications and possibilities of the new device [10].

The concept of inertial amplification was also utilised by Yilmaz and Kikuchi [11]. They employed periodically repeated masses and levers to create multiple anti-resonance frequencies and corresponding wide attenuation frequency bands. In the paper, two different configurations of vibration isolators were investigated, showing possibilities for effective vibration attenuation, i.e. deep and wide bands, in the low-frequency range. Further works published by Yilmaz and collaborators [12, 13, 14] show other applications of the inertial vibration isolation concept. As an example, a two-dimensional lattice with an embedded periodically repeated inertial mechanism is analysed in 
Yilmaz's paper [12], where the potential of the inertial amplification is presented.

Based on Yilmaz's geometric design [12], the inertial amplification mechanism concept has been utilised by Frandsen and collaborators in [15] in order to create low-frequency wide band gaps for longitudinal waves in a continuous $1 \mathrm{D}$ rod. The mechanism is attached periodically to the surface of the host rod, keeping the structural functionality intact. The results illustrate that the mechanism can indeed be used to attenuate longitudinal vibrations by creating wide and deep band gaps at low frequency with a relatively small added mass fraction. The promising results combined with practical capabilities of the mechanism have encouraged further research in this area.

In this paper a system similar to the one described in [15] is considered, however, we will focus on the use of the inertial amplification mechanism to attenuate bending vibrations, since these are of fundamental importance in many engineering structures. In the paper [12] the transverse and longitudinal vibrations have been considered simultaneously indicating the the mechanism can work for vibrations in multiple dimensions. Hence, we investigate the potential for the mechanism to attenuate bending vibrations of a continuous host beam, which will potentially allow us to protect the structure against both longitudinal and transverse vibrations.

\section{Modeling}

A slender elastic beam structure with an inertial amplification mechanism attached to the top of the beam, as illustrated in Fig. 1, is considered. The mechanism is connected to the top surface of the beam via hinges and has a moment-free connection at the top. This can physically be approximated by housing and ball bearings, whereby the assumptions are not too restrictive. Furthermore, we assume that the connecting bars are rigid and massless. The additional mass attached at the top generates the inertial force. The elastic beam has the following parame-

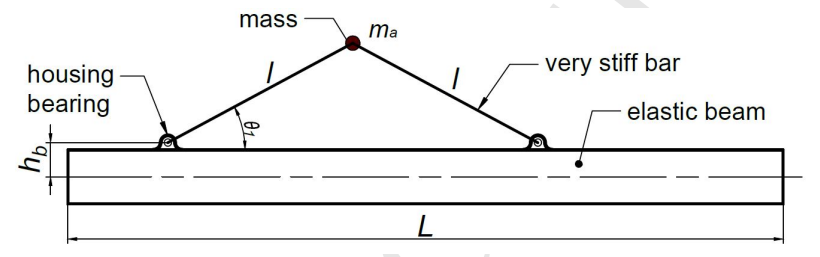

Figure 1: The elastic beam with attached inertial amplification (IA) mechanism.

ters: Young modulus $E$, mass density $\rho$, thickness $t_{b}$, width $w_{b}$ and length $L$. The mechanism is described by the amplification mass $m_{a}$, initial angle $\theta_{1}$ and distance between natural axis of the beam and center of the bearing $h_{b}$.

\subsection{Mechanism kinematics}

The displacement of the attached mass is denoted by $x_{m}$ and $y_{m}$ in the horizontal and vertical direction, respectively. Based on the kinematics of the amplification mechanism, its motion can be expressed by the vertical and longitudinal motion at the attachment points, which is expressed in terms of vertical motion and rotation of the beam center line respectively: $y_{1}, y_{2}, \varphi_{1}$, $\varphi_{2}$ as well as the initial angle $\theta_{1}$ and distance $h_{b}$ (see Fig. 2). The equations for the displacement components are derived by geometric considerations presented in Appendix A. The linearised form of the equations is shown in Eq. (1a-b):

$$
\begin{aligned}
& y_{m}=\frac{1}{2}\left(y_{2}+y_{1}\right)-\frac{1}{2} h_{b}\left(\varphi_{1}-\varphi_{2}\right) \cot \left(\theta_{1}\right) \\
& x_{m}=\frac{1}{2}\left(-y_{2}+y_{1}\right) \tan \left(\theta_{1}\right)-\frac{1}{2} h_{b}\left(\varphi_{1}+\varphi_{2}\right)
\end{aligned}
$$

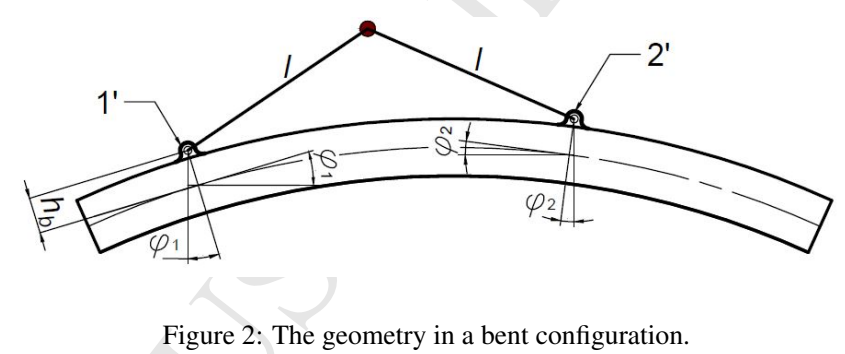

\subsection{Mechanism equation of motion}

The equation of motion for the beam with the attached mechanism is derived in two steps. First, we relate the acceleration of the mass to the forces and moments transmitted at the attachments points using Lagrange's equation for the isolated inertial amplification mechanism, as illustrated in Fig. 3, where the dis-

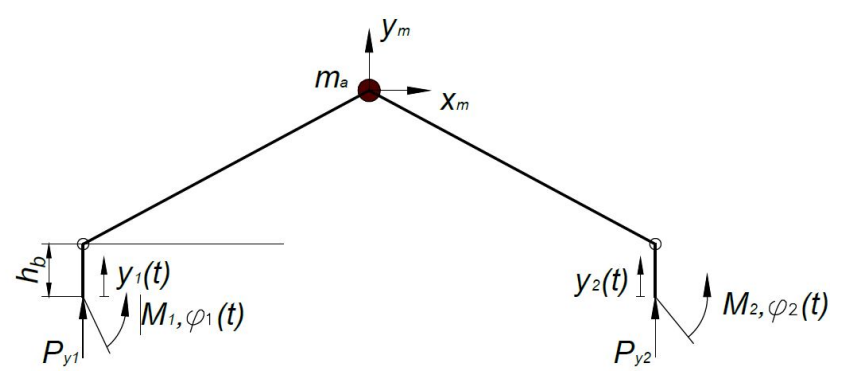

Figure 3: Mechanism with attachment point forces and moments.

tance $h_{b}$ represents the distance between the centreline of the beam and the attachment of the mechanism on the surface of the beam. The Lagrangian is given by:

$$
\frac{d}{d t}\left(\frac{\partial L}{\partial \dot{q}_{i}}\right)-\frac{\partial L}{\partial q_{i}}=\frac{\partial W}{\partial q_{i}}
$$

where the Lagrangian $L$ represents the difference between kinetic and potential energy, $L=T-V$ and the work $W$ depends on the applied forces. The kinetic energy of the system is expressed in terms of generalized coordinates $q_{i}$, in this case: $y_{1}(t), y_{2}(t), \varphi_{1}(t)$ and $\varphi_{2}(t)$ [16]. Motion of the ideal inertial amplification mechanism only generates kinetic energy, given by:

$$
T=\frac{1}{2} m_{a}\left(\dot{y}_{m}^{2}+\dot{x}_{m}^{2}\right)
$$


where $m_{a}$ is the attached mass at the top of the mechanism. We can now derive the corresponding relations and express these in matrix form.

$$
\left[\begin{array}{cccc}
m_{1} & -m_{2} & m_{3} & m_{4} \\
-m_{4} & m_{5} & -m_{4} & m_{6} \\
m_{3} & -m_{4} & m_{1} & m_{2} \\
m_{4} & m_{6} & m_{4} & m_{5}
\end{array}\right]\left[\begin{array}{c}
\ddot{y}_{1} \\
\ddot{\varphi}_{1} \\
\ddot{y}_{2} \\
\ddot{\varphi}_{2}
\end{array}\right]=\left[\begin{array}{c}
P_{1 y} \\
M_{1} \\
P_{2 y} \\
M_{2}
\end{array}\right]
$$

where the factors $m_{i}$ are effective masses of the inertial amplification mechanism and given as:

$$
\begin{aligned}
& m_{1}=\frac{1}{4} m_{a}\left(1+\tan \left(\theta_{1}\right)^{2}\right) \\
& m_{2}=\frac{1}{4} m_{a} h_{b}\left(\cot \left(\theta_{1}\right)+\tan \left(\theta_{1}\right)\right) \\
& m_{3}=\frac{1}{4} m_{a}\left(1-\tan \left(\theta_{1}\right)^{2}\right) \\
& m_{4}=\frac{1}{4} m_{a} h_{b}\left(\cot \left(\theta_{1}\right)-\tan \left(\theta_{1}\right)\right) \\
& m_{5}=\frac{1}{4} m_{a} h_{b}^{2}\left(1+\cot \left(\theta_{1}\right)^{2}\right) \\
& m_{6}=\frac{1}{4} m_{a} h_{b}^{2}\left(1-\cot \left(\theta_{1}\right)^{2}\right)
\end{aligned}
$$

\subsection{System equations of motion}

The slender elastic beam is modelled using standard Bernoulli-Euler beam theory. The inclusion of the mechanism in the model is most conveniently done directly in a finite element model of the beam where we can apply the forces and moments generated at the mechanism attachment points directly as nodal loads. We use a standard one-dimensional beam element with 4 degrees of freedom (d.o.f) - lateral translations: $u_{1}, u_{2}$ and rotations $\psi_{1}$ and $\psi_{2}$, where the rotation vector is normal to $x y$-plane. Axial dofs are neglected. The equations of motion of the discretized system then become:

$$
\mathbf{M u ̈}+\mathbf{K u}=\mathbf{F}-\mathbf{v}_{1} P_{1 y}-\mathbf{v}_{2} M_{1}-\mathbf{v}_{3} P_{2 y}-\mathbf{v}_{4} M_{2}
$$

where $\mathbf{u}=\left\{u_{1} \psi_{1} \ldots u_{n} \psi_{n}\right\}^{T}$ is the global vector of beam nodal dof's and $\mathbf{v}_{i}=[0 \ldots 1 \ldots]^{T}$ denotes the index vectors, introduced to apply the mechanism forces at the appropriate dof's. The matrices $\mathbf{M}$ and $\mathbf{K}$ represent the standard mass and stiffness matrices for the beam, respectively. External forces are contained in $\mathbf{F}$. Considering the mechanism equation of motion Eq. (4), the generalized displacements $y_{1}, \varphi_{1}, y_{2}$ and $\varphi_{2}$ can be expressed as $y_{1}=\mathbf{v}_{1} \mathbf{u}, \varphi_{1}=\mathbf{v}_{2} \mathbf{u}, y_{2}=\mathbf{v}_{3} \mathbf{u}, \varphi_{2}=\mathbf{v}_{4} \mathbf{u}$ and introducing the $\mathbf{v}=\left[\begin{array}{lll}\mathbf{v}_{1} & \mathbf{v}_{2} & \mathbf{v}_{3} \\ \mathbf{v}_{4}\end{array}\right]$, whereby Eq. (6) becomes

$$
\begin{aligned}
& \tilde{\mathbf{M}} \ddot{\mathbf{u}}+\mathbf{K u}=\mathbf{F} \\
& \tilde{\mathbf{M}}=\mathbf{M}+\mathbf{v M}_{\mathbf{I A}} \mathbf{v}^{\mathbf{T}}
\end{aligned}
$$

where the inertial amplification matrix $\mathbf{M}_{\mathbf{I A}}$ is the mass matrix from Eq. (4). The contribution from the mechanism is included in the appropriate degree of freedom by the influence vector $\mathbf{v}$.

We will study the steady-state vibrations of the beam when subjected to a time-harmonic force $\mathbf{F}=\mathbf{F}_{0} \cos (\omega t)$ so that the resulting equations are:

$$
\left(-\omega^{2} \tilde{\mathbf{M}}+\mathbf{K}\right) \mathbf{u}_{0}=\mathbf{F}_{0}
$$

where $\mathbf{u}_{0}$ is the corresponding vibration amplitude vector.

\section{Analysis}

In this section the effects of attaching the IA mechanism to a steel beam with the parameters given in Tab. 1 is illustrated. Fig. 4 shows the analysed system: a free-free beam with the IA

Table 1: Basic beam properties.

\begin{tabular}{lllll}
\hline \hline$L[\mathrm{~m}]$ & $w_{b}[\mathrm{~m}]$ & $t_{b}[\mathrm{~m}]$ & $E[\mathrm{~Pa}]$ & $\rho\left[\mathrm{kg} / \mathrm{m}^{3}\right]$ \\
\hline 0.5 & 0.05 & 0.005 & $2.1 \times 10^{11}$ & 7850 \\
\hline \hline
\end{tabular}

mechanism attached is subjected to a vertical, time-harmonic force in one end. The response is evaluated as the vertical vibration amplitude at the other beam end.

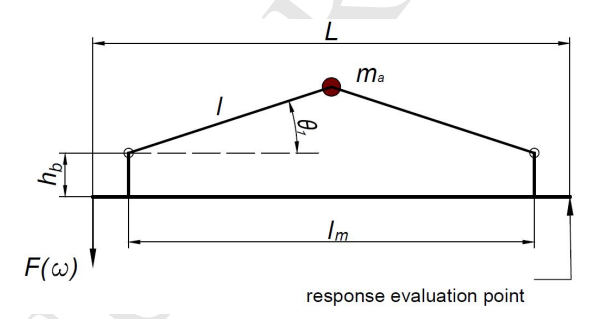

Figure 4: Indication of key system parameters.

Fig. 5 shows the FRF of the vertical beam tip response for the IA parameters seen in Tab. 2 , where $M_{b}$ denotes the total mass of the host beam. The presence of anti-resonances is noted as sharp dips in the FRF. Especially the presence of two neighbouring anti-resonances without an in-between resonance peak is noteworthy, since this increases the bandwidth of the attenuation effect significantly. This effect has previously been demonstrated for other IA devices, e.g., in [12, 9, 15]. Next,

Table 2: IA parameters corresponding to the FRFs in Fig. 5.

\begin{tabular}{lllr}
\hline$\theta_{1}[\mathrm{rad}]$ & $m_{a} / M_{b}[\%]$ & $l_{m} / L[\mathrm{~m}]$ & $h_{b}[\mathrm{~m}]$ \\
\hline$\pi / 24.7$ & 20 & 1 & 0.013 \\
\hline \hline
\end{tabular}

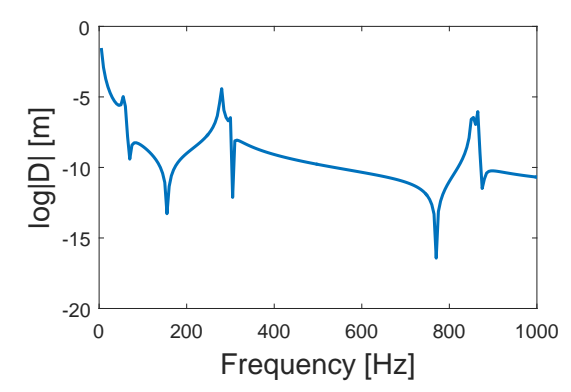

Figure 5: Sample FRF plot illustrating the basic behaviour including double anti-resonance dips.

we make a numerical investigation of the system performance when the mechanism parameters are varied using the simple model derived in the previous section. We will later compare the results using the idealized conditions (e.g. massless, rigid 
mechanism bars) to a 3D ANSYS model with realistic material parameters.

\subsection{Effect of added mass}

Consider the beam with the parameters in Tab. 1 and a mechanism with dimensions given in Tab. 3. Increasing the amplifi-

Table 3: IA parameters corresponding to the FRFs in Fig. 6.

\begin{tabular}{lcr}
\hline \hline$\theta_{1}[\mathrm{rad}]$ & $l_{m} / L$ & $h_{b}[\mathrm{~m}]$ \\
\hline$\pi / 21$ & 0.85 & 0.013 \\
\hline \hline
\end{tabular}

cation mass is a simple way to boost the inertia force generated by the mechanism, hence it is expected that this will extend gap width and decrease the affected frequency as well. The plots of the FRF's for different values of the mass ratio $\mu=m_{a} / M_{b}$ are illustrated in Fig. 6.
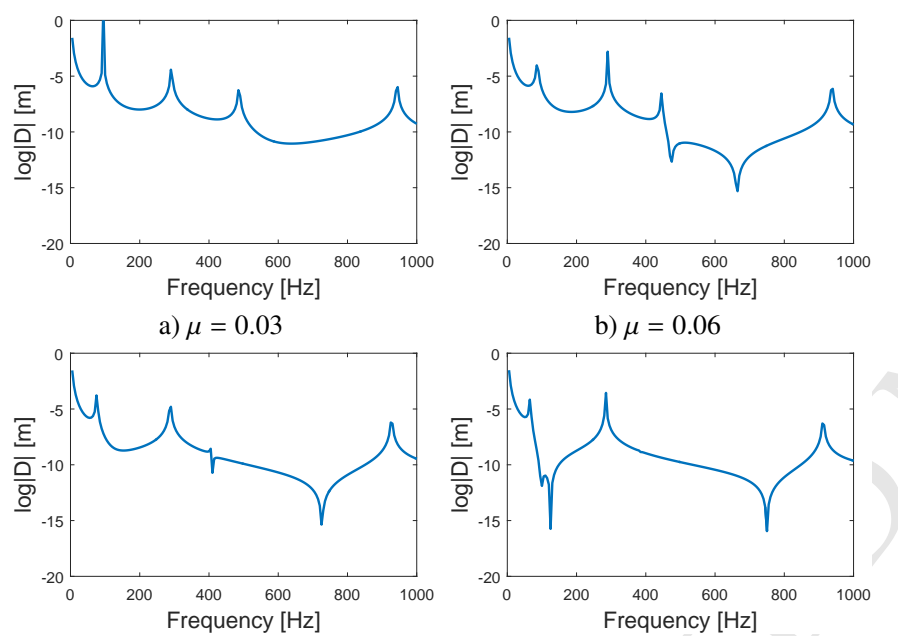

c) $\mu=0.12$

d) $\mu=0.21$

Figure 6: Effect of varying the mass ratio $\mu$.

It can be seen in Fig. 6 that the effect of $\mu$ on the FRF is non-trivial, but generally double-dip anti-resonances are created at lower frequency for larger $\mu$ leading to corresponding frequency ranges with vibration attenuation. For $\mu=0.06$ large vibration attenuation is found between the third and fourth resonance frequency in the range from approximately $450-800$ [Hz], whereas for $\mu=0.21$ a frequency range between 100-250 $[\mathrm{Hz}]$ with strong attenuation is created between the first and second resonance. The non-trivial dependency on $\mu$ is highlighted by the more limited effect noted for the intermediate value of $\mu=0.12$, however it should be noted that the third resonance peak is practically removed.

\subsection{Effect of mechanism width}

The location of the attachment points has a significant impact on the amplification effectiveness. The system is analysed for four cases with different relative length of the mechanism $\bar{l}=l_{m} / L$, and the general IA parameters given in Tab. 4 .
Table 4: IA parameters corresponding to the FRFs in Fig. 8.

\begin{tabular}{llr}
\hline \hline$\theta_{1}[\mathrm{rad}]$ & $m_{a} / M_{b}[\%]$ & $h_{b}[\mathrm{~m}]$ \\
\hline$\pi / 21$ & 20 & 0.013 \\
\hline \hline
\end{tabular}

The inertial amplification effect is heavily influenced by the rotation at the attachment points. This can be seen in Eq. (4)(5), where the dominating terms are related to cross-section rotation. Considering the mechanism being originally developed for longitudinal motion, this is not surprising, since crosssection rotation leads to longitudinal motion of the outermost fiber of the beam. Hence, maximal (out of phase) rotation at the two attachment points will maximize efficiency of the mechanism. Furthermore, the attachment points determine the length of the mechanism $l_{m}$, which also has an impact on the performance metrics, i.e. the bandwidth and level of attenuation. It is expected that the effectiveness generally increases with a longer mechanism length due to the increased lever-arm for the inertial force. This is illustrated in Fig. 7 that shows the first bending mode of vibration for a freely suspended beam with an attached symmetric mechanism. Here it is seen how the distance $l_{m}$ functions as a lever arm for the forces $\mathbf{P}$ which together with the moments $\mathbf{M}$ act against the elastic forces in the beam.

Fig. 8 shows the FRF for the systems with attached symmetric mechanisms with different lengths $l_{m}$.

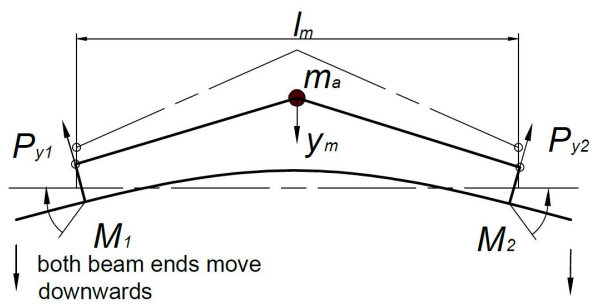

Figure 7: Illustration of the system near the fundamental mode of vibration.
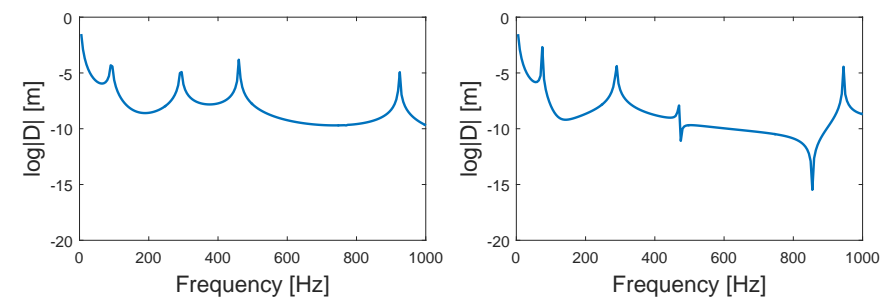

d) $\bar{l}=0.55$

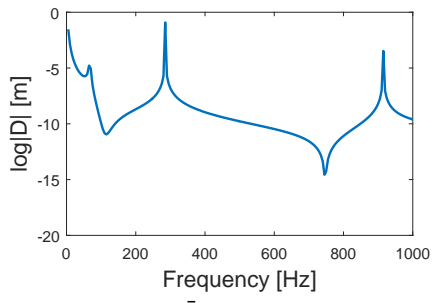

c) $\bar{l}=0.75$

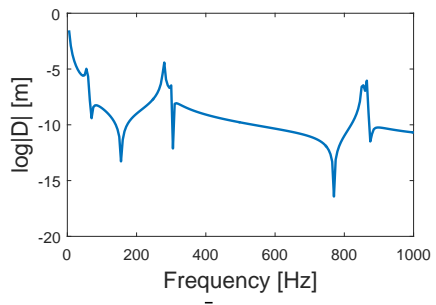

b) $\bar{l}=0.85$

a) $\bar{l}=1$

Figure 8: FRFs for varying internal length $\bar{l}=l_{m} / L$ 
It can be seen, that the attenuation efficiency of the system increases with increasing length ratio $\bar{l}$. For $\bar{l}=1$ and $\bar{l}=0.85$, the low-frequency anti-resonances are observed, which disappear with decreasing relative length. This behaviour is in line with above expectation, and shows the importance of the lever arm.

For all above analysed cases it is observed that broadband gaps associated with the double-dip anti-resonances occur after odd-numbered vibration modes. This behaviour is related to the shape of the vibration form and the symmetric configuration of the system with the additional mass located at midpoint. For even-numbered modes, the attachment points of the mechanism rotate in phase, which leads to insignificant motion of the additional mass, hence, no or small inertial force is generated.

\subsection{Effect of bearing height}

The bearing height, $h_{b}$, also has significant influence on the attenuation features of the inertial amplification mechanism. It works as a lever which transforms the internal force of the mechanism mass to moments at the attachment nodes. Thus with increasing distance $h_{b}$ the moment increases too. Fig. 9 presents the FRF for the system with the parameters of the IA mechanism given in Tab. 5.

Table 5: IA parameters corresponding to the FRFs in Fig. 9.

\begin{tabular}{llr}
\hline \hline$\theta_{1}[\mathrm{rad}]$ & $m_{a} / M_{b}[\%]$ & $l_{m} / L[\mathrm{~m}]$ \\
\hline$\pi / 21$ & 11 & 0.85 \\
\hline \hline
\end{tabular}

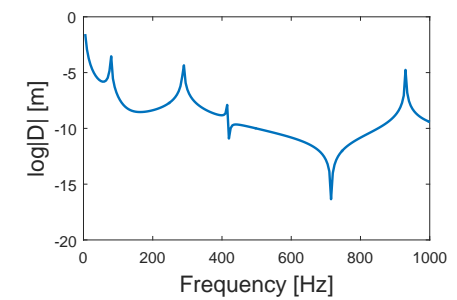

a) $h_{b}=0.013[\mathrm{~m}]$

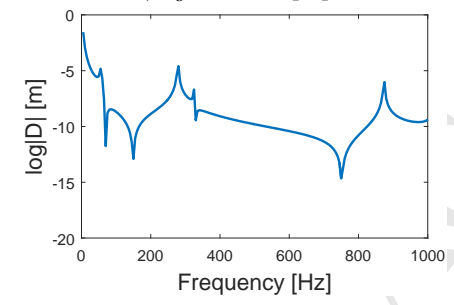

c) $h_{b}=0.023[\mathrm{~m}]$

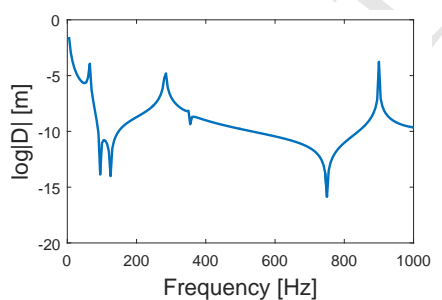

b) $h_{b}=0.018[\mathrm{~m}]$

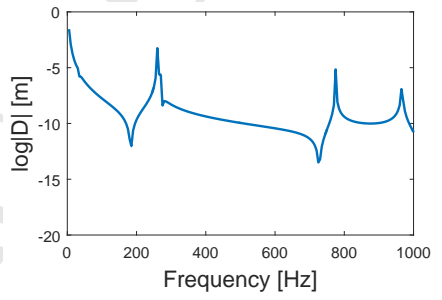

d) $h_{b}=0.035[\mathrm{~m}]$
Figure 9: Comparison different values of bearing height parameter $h_{b}$.

When comparing Fig. 9a) and Fig. 9b) the effect of the enhanced inertial force due to increased height $h_{b}$ is observed, i.e. a new double-peak dip has appeared and the two existing dips are further separated. Further increasing the value of parameter $h_{b}$ improves performance but does not produce the significant effects seen for the initial increment.

\subsection{Comparison to a standard vibration absorber}

The performance of the designed mechanism with the parameters given in Tab. 6 is now compared to a standard vibration absorber with comparable parameters. Both systems are attached to the beam with parameters presented in Tab. 7 The

Table 6: IA parameters corresponding to the FRFs in Fig. 12.

\begin{tabular}{lllr}
\hline \hline$\theta_{1}[\mathrm{rad}]$ & $m_{a} / M_{b}[\%]$ & $l_{m} / L$ & $h_{b}[\mathrm{~m}]$ \\
\hline$\pi / 72$ & 8.5 & 0.87 & 0.023 \\
\hline \hline
\end{tabular}

Table 7: Beam properties corresponding to the FRFs in Fig. 12.

\begin{tabular}{lllll}
\hline \hline$L[\mathrm{~mm}]$ & $w_{b}[\mathrm{~mm}]$ & $t_{b}[\mathrm{~mm}]$ & $E[\mathrm{~Pa}]$ & $\rho\left[\mathrm{kg} / \mathrm{m}^{3}\right]$ \\
\hline 0.9 & 0.05 & 0.005 & $2.1 \times 10^{11}$ & 7875 \\
\hline \hline
\end{tabular}

performance of the standard system is presented for two tuning cases. The first is when the local resonator generates the antiresonance frequency between the $1^{\text {st }}$ and $2^{\text {nd }}$ mode. For the second case, the system is tuned to get the same anti-resonance in $225[\mathrm{~Hz}]$ as for the IA. Both cases with the parameters given in Figs. 10 and 11. The FRFs for two cases are presented in

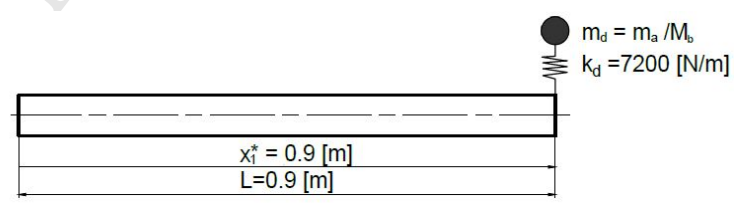

Figure 10: Location of the local resonator on the beam- case no. 1.

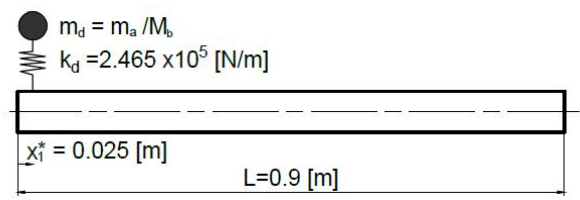

Figure 11: Location of the local resonator on the beam- case no. 2.

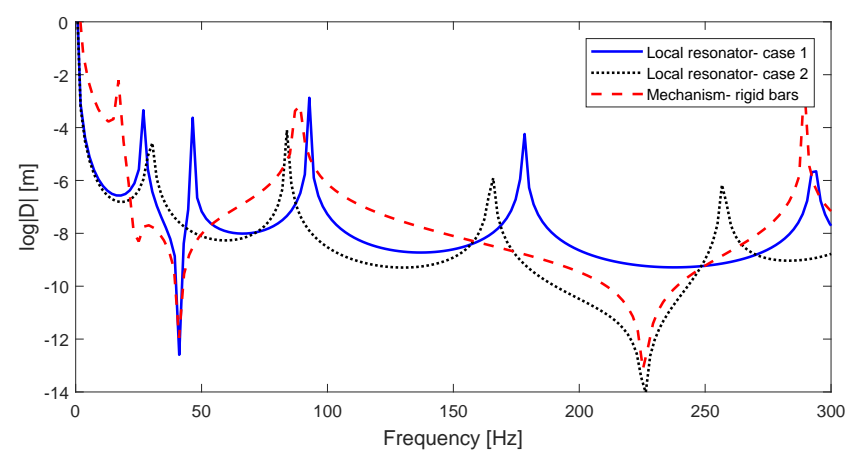

Figure 12: Comparison FRFs of the local resonator to the inertial amplifier.

Fig. 12. It is seen that the standard dynamic absorber is able to create the gap at low-frequency, however, with a narrower 
frequency range of attenuation. This is even more evident in case no. 2 (Fig. 12), where we can observe a wide gap generated by the IA, extended between $90-290$ [Hz]. Conversely, the local resonator gap extends between 160 to 260 [Hz], i.e., the range is $50 \%$ narrower. Apart from its higher efficiency, the proposed mechanism contains multiple parameters that can be tuned, providing a larger design flexibility, along with the fact that the IA attenuates multiple modes.

Thus, for this idealized example the inertial amplification mechanism provides improved efficiency, i.e. wide and multiple gaps in the FRF, when compared to the classical local resonance system. This supports previous results and conclusions $[15,11,17]$.

\section{Finite element validation}

A model of the inertial amplification mechanism is implemented in the commercial FE-software Ansys. The host beam as well as the bars of the system are modelled by threedimensional beam elements - BEAM188. The two-noded element is based on Timoshenko beam theory which includes shear deformations. The bars are connected with the beam using revolution joints in order to obtain rotationally free connections. The distance $h_{b}$ which corresponds to the dimension of a housing bearing is modelled as a very stiff and massless beam in order to avoid local bending. The masses of elements such as: ball bearing, hinges and housing bearing are implemented as the point masses at corresponding nodes [18].

For all following cases a time-harmonic excitation force (with an arbitrary amplitude set to $F=100 \mathrm{~N}$ ) is applied at the first node (at the left beam end) in the vertical direction and the response of the system is measured at the last node (the vertical vibration amplitude at the right beam end).

The mechanism is always attached with the widest possible distance $l_{m} / L=0.87$ in the numerical analysis. This particular value is limited by the restrictions related to the physical model of the system, presented in the next section. The widely attached mechanism has been shown to affect the first bending mode the most, whereby the lowest anti-resonances can be obtained. The host beam has the material parameters given in Tab. 7. When Young's modulus of the connecting bars is set to a realistic values, their flexibility becomes influential. Fig. 13 illustrates the FRFs for both rigid and realistic connecting bars, for the mechanism parameters in Tab. 8. Rigid bars are obtained by setting $E_{b}=1 \times 10^{20}[\mathrm{~Pa}]$.

Table 8: IA mechanism parameters - case no. 1

\begin{tabular}{lllr}
\hline \hline$\theta_{1}[\mathrm{rad}]$ & $m_{a} / M_{b}[\%]$ & $l_{m} / L$ & $h_{b}[\mathrm{~m}]$ \\
\hline$\pi / 44$ & 8.5 & 0.87 & 0.012 \\
\hline \hline
\end{tabular}

Fig. 13 shows that for the case with rigid bars, two wide gaps are obtained: the first double-dip gap is approximately within the range $130-290[\mathrm{~Hz}]$ and the second gap is located between $310-590[\mathrm{~Hz}]$. When using true physical bar parameters the high-frequency gap disappears but the first remains and displays

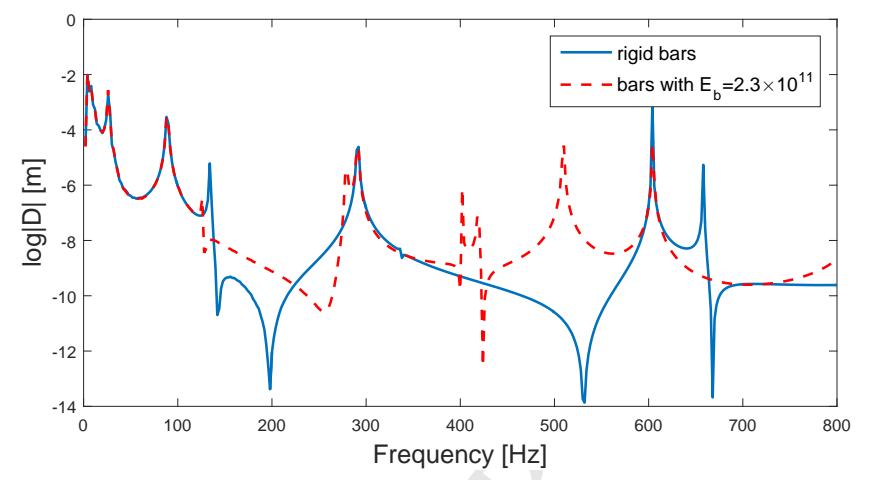

Figure 13: FRF's of the refined model, $h_{b}=0.012[\mathrm{~m}], \theta_{1}=\pi / 44[\mathrm{rad}]$ - case no. 2 .

two dips in the range $120-270[\mathrm{~Hz}]$ and generally even though discrepancies between the FRFs are present, similar trends are observed. Fig. 14 illustrates the deformation shapes of the system at a number of specific frequencies in order to illustrate the reasons for the discrepancies.

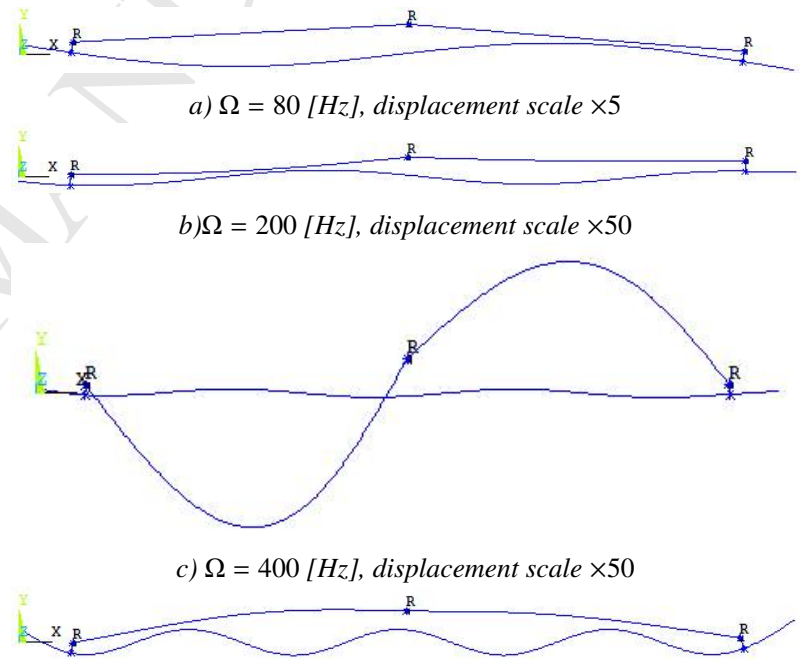

d) $\Omega=790[\mathrm{~Hz}]$, displacement scale $\times 300$

Figure 14: Deflection of the mechanism with non-rigid bars.

Comparing the plots in Fig. 14 it can be clearly seen that the vibration amplitude of the bars increases significantly for 400 $[\mathrm{Hz}]$, corresponding to their fundamental natural frequency, whereby the system actually works by local resonance. This results in the observed resonance peak in the FRF for the system with physical parameters in Fig. 13.

This phenomenon is accurately described in Yilmaz and Hulbert's paper [13], which states that the inertial amplification effect will be dominating if the IA-antiresonance is below the local resonance frequency of the mechanism. The authors show this by changing the stiffness of their IA and analysing the width and depth of the inertial amplification induced gaps.

The analysis of the system focuses on low-frequency gaps, between the $1^{s t}-2^{\text {nd }}$ and $2^{\text {nd }}-3^{r d}$ modes which are below 400 $[\mathrm{Hz}]$, thus the local resonance of the mechanism is not an issue 
in the most analysed cases.

The results obtained by Ansys are compared with the FRFs obtained using the simple model. It is expected that profiles of the curves should match closely for the cases with rigid bars, but also with the physical parameters of the bars included, the corresponding FRF's should show similar trend in low frequency range. Hence, the plots are limited up to $300[\mathrm{~Hz}]$ and illustrated in Figs. 15 through 17. Moreover, in the graphs, the natural frequencies of the pure beam are marked by the gray dotted lines in order to see the impact of the mechanism on the resonance peaks of the pure beam. Analyses are performed for three different setups of the attached mechanism, with the parameters given in Tab. 9 .

Table 9: IA parameters corresponding to the FRFs in Fig. 15-17.

\begin{tabular}{llllr}
\hline \hline & $\theta_{1}[\mathrm{rad}]$ & $m_{a} / M_{b}[\%]$ & $l_{m} / L$ & $h_{b}[\mathrm{~m}]$ \\
\hline $1^{\text {st }}$ case & $\pi / 44$ & 8.5 & 0.87 & 0.012 \\
$2^{\text {nd }}$ case & $\pi / 72$ & 8.5 & 0.87 & 0.023 \\
$3^{\text {rd }}$ case & $\pi / 33$ & 8.5 & 0.87 & 0.023 \\
\hline \hline
\end{tabular}

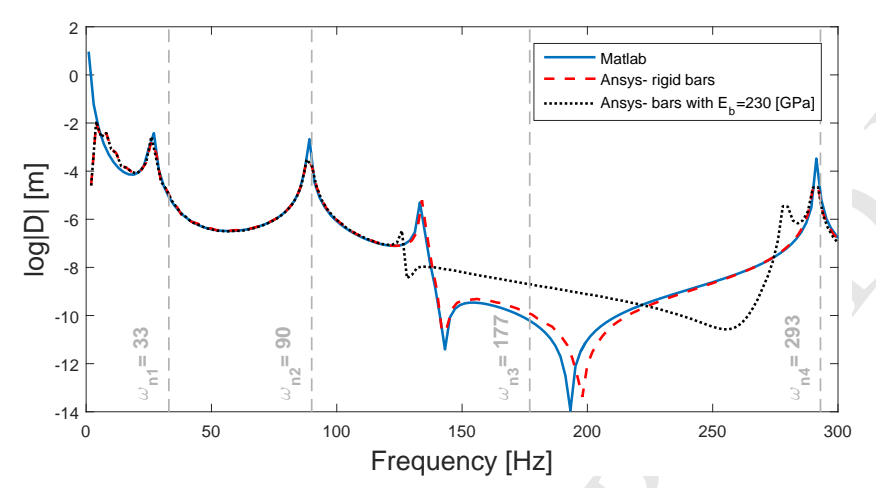

Figure 15: Comparison between MatLab and ANsys solutions: $h_{b}=0.012$ [m], $\theta=\pi / 44[\mathrm{rad}]$.

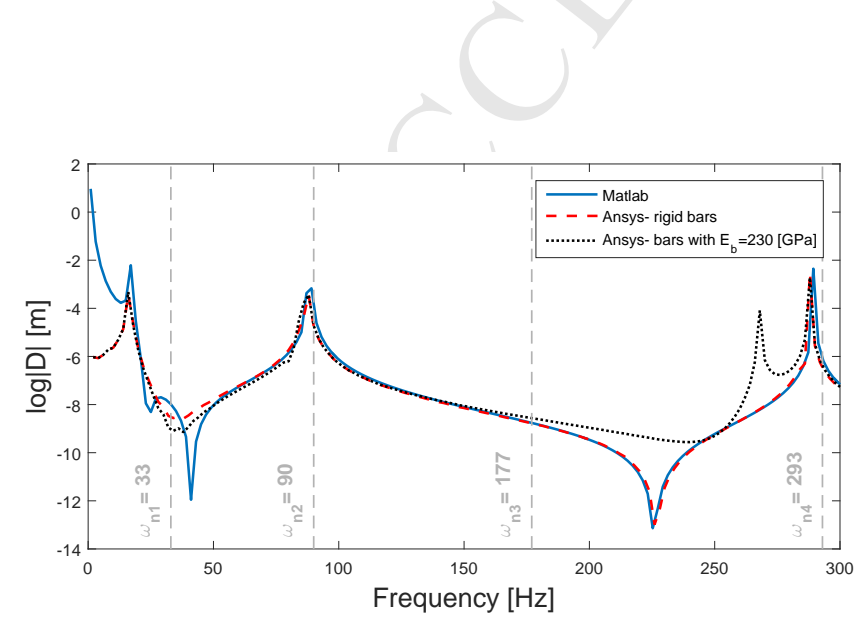

Figure 16: Comparison between MatLAB and ANsys solutions: $h_{b}=0.023$ [m], $\theta=\pi / 72$ [rad].

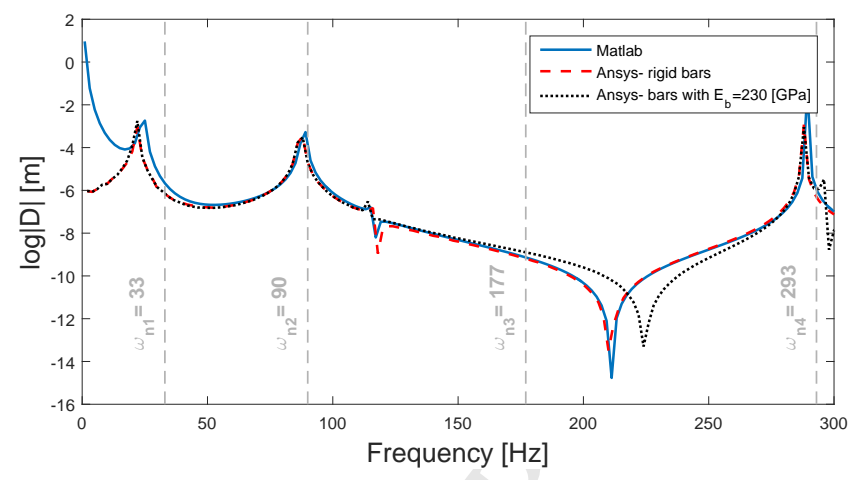

Figure 17: Comparison between MatLAB and Ansys solutions: $h_{b}=0.023[\mathrm{~m}]$, $\theta=\pi / 33[\mathrm{rad}]$.

It is noted that with rigid bars the results obtained by both methods match well and justifies the use of the simple model in the initial design phase for the experiments. Considering the mechanism with real material parameters, it can be seen that the response curve in Fig. 17 has nearly the same profile as the one for the system with the rigid bars. In Figs. 15 and 16 for systems with $E_{b}=2.3 \times 10^{11}[\mathrm{~Pa}]$, the gaps are found in almost identical frequency ranges in comparison to the response curves for the mechanism with the rigid bars. This further supports the argument for using the simple model in the initial design phase. It is also observed in Fig. 15 that due to the vibration of the bars the sharp dips do not occur for the mechanism with real material parameters.

In Fig. 16 around 33 [Hz], FRF's obtained by Ansys and the simple model show somewhat different trends. The antiresonance dips in the simple model function are sharp with a significant distance between them in comparison to a single, rounded dip seen in the Ansys simulation. This can be explained by the axial deformations present in FE model, but omitted in the simple model. Considering both attachment points of the mechanism, the magnitude of the axial deformation at the first one is about 6 times smaller than the transverse deformation. For the second point, this proportion increases to 70. As it can be seen, the deformations in the two directions have different orders of a magnitude, but still the axial component might affect the results since the energy associated with the axial direction requires much less deformation to be significant in comparison to the transverse deformation. Furthermore, this phenomenon is amplified for the small angle $\theta_{1}$ as in this particular case, for which the mechanism generates a large axial force along the beam.

\section{Experimental setup}

Based on the parameter analysis of the mechanism, a $3 D$ model of the system is created in a CAD software, illustrated Fig. 18. System parameters are chosen to create large vibration attenuation in the challenging low-frequency range with a moderate amount of added mass. The prior analysis has shown that low-frequency gaps are observed for mass ratio $\mu=0.06$ thus, the restriction is made to maintain a ratio between $\mu=0.06-0.15$. The designed test rig, illustrated in 


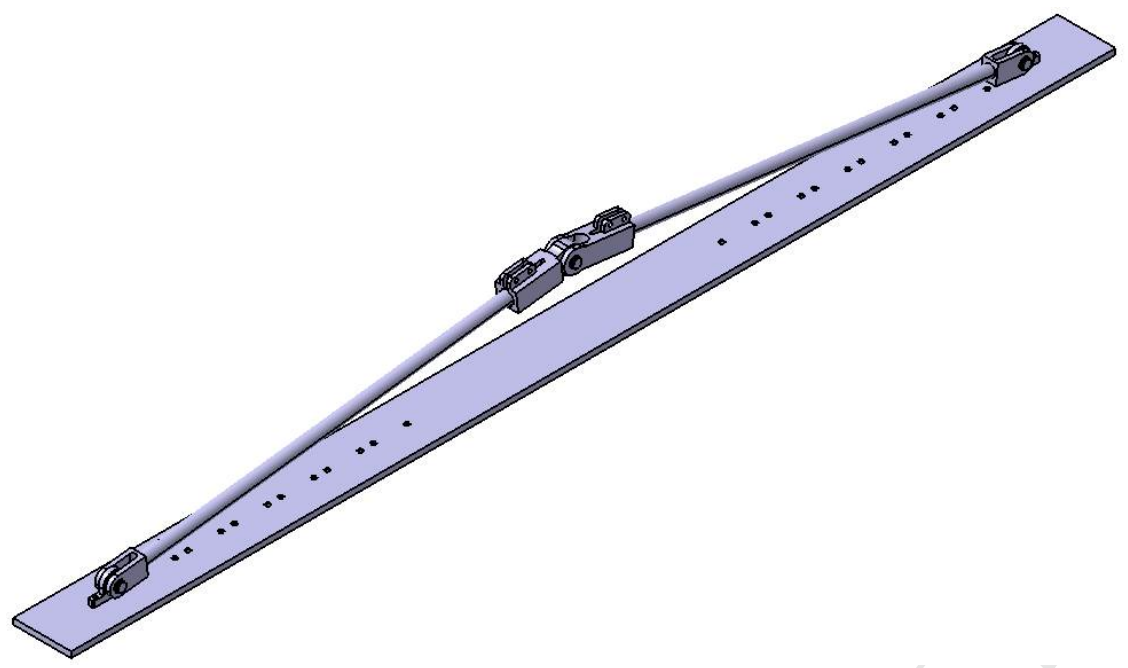

Figure 18: Inertial amplification mechanism - isometric view.

Fig. 19, is used for experimental modal analysis. The beam is excited by a modal hammer at one end of the beam and the response is measured by an accelerometer at the second end. The impulse excitation allows to examine the response in a wide frequency range. This type of test is simple and fast, therefore it is possible to investigate the mechanism for many different configurations. As it can be seen in Fig. 19 the beam with the attached mechanism hangs on two rubber bands. This attachment imitates the free-free boundary conditions. The accelerometer is mounted beneath the beam at the measurement point and the excitation force is applied on the top surface of the beam. The tests are analysed using Brüel \& Kjaer digital frequency analyser [19]. The hardware schematics is illustrated Fig. D.30.

The four FRF's in the previous chapter, obtained by numerical analysis in Ansys, are now compared to relevant experimental results in Figs. 20 through 22. Setup data are presented in Tab. 9.

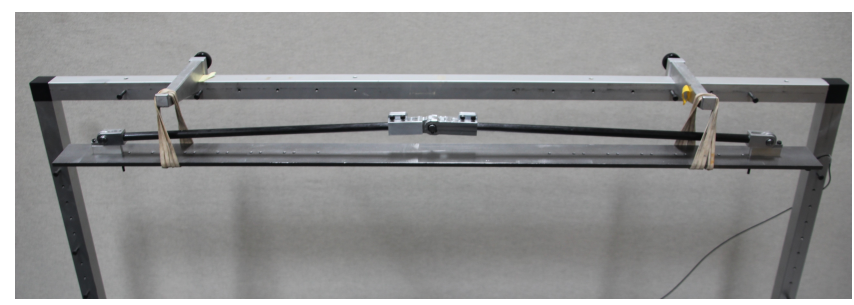

Figure 19: Experimental test rig.

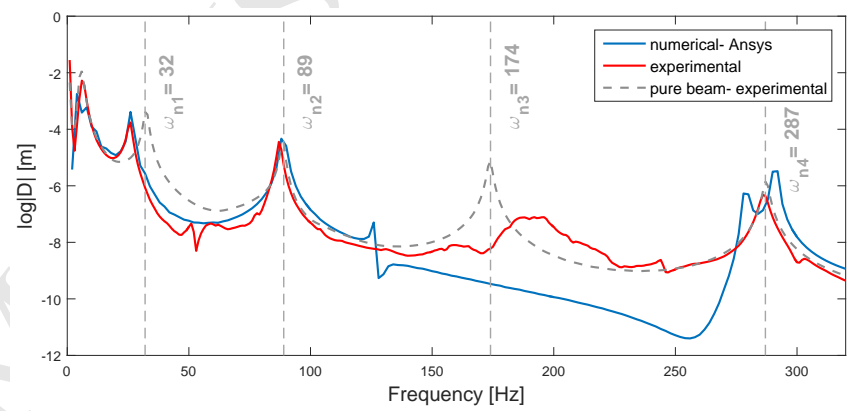

Figure 20: FRFs for $1^{s t}$ case.

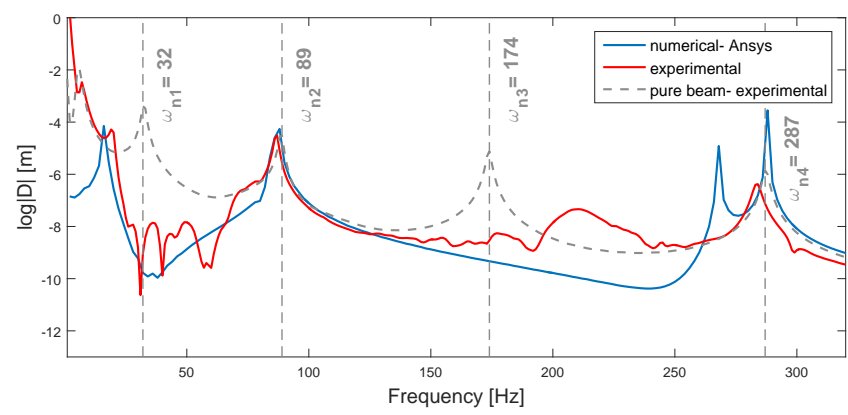

Figure 21: FRFs for $2^{\text {nd }}$ case.

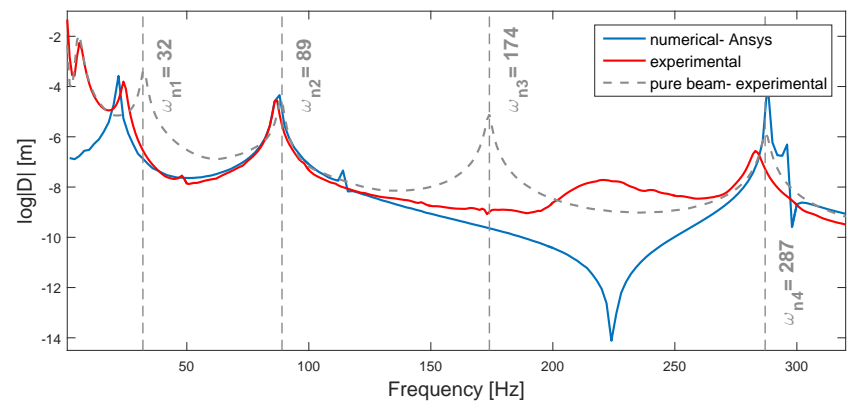

Figure 22: FRFs for $3^{\text {rd }}$ case. 
It is noted that for all investigated cases in the frequency range $0-20[\mathrm{~Hz}]$ the differences between numerical and experimental curves are significant. The experimental FRFs have a number of resonance peaks within this range, which is attributed to the resonance frequencies of the rubber bands.

As demonstrated in Figs. 20-22, discrepancies between the numerical and experimental FRFs are primarily found at the anti-resonance frequencies. The experimental curves mostly present a rather flat behaviour in the attenuated zones, as opposed to the dips found in the numerical results. These discrepancies might be due to measurement inaccuracies or nonlinear effects such as backlash and friction, since sliding bearings are used in the inertial mechanism. Furthermore, due to the system construction, the backlash is additionally amplified for small angle $\theta_{1}$. For the all considered set-ups, this angle is relatively small, what might lead to observed discrepancies.

The desirable effect of the inertial amplification mechanism is most clearly illustrated in Fig. 21. Consider the blue numerical and red experimental curve. For the numerical FRF the double-dip gap is observed in range $20-90$ [Hz]. The similar phenomena can be seen in the experiments, which shows multiple dips in the same range. This indicates efficient operation of the mechanism and its ability to create gaps formed by multiple anti-resonance dips. In both cases the first mode is shifted to the left, hence the gap is relatively wide $(70[\mathrm{~Hz}])$. Moreover, the $3^{\text {rd }}$ resonance peak for experimental case is moved to the right but not as significantly as predicted by the numerical simulation.

\subsection{Mechanism parameters variation}

The experimental setup is used to demonstrate the effect of the important parameters of the system, i.e., the neutral axis distance $h_{b}$, the initial angle $\theta_{1}$ and the location of the attachment points. Due to the construction of the mechanism, it was impracticable to vary each parameter individually while keeping all others constant, e.g., changing the internal length of the mechanisms affects the angle $\theta_{1}$. During the experiment, the parameter $h_{1}$ (see Fig. 23), was used as a control parameter in order to keep the different parameters as constant as possible. The variation in added mass due to different lengths of the carbon connecting rods was deemed insignificant and disregarded.

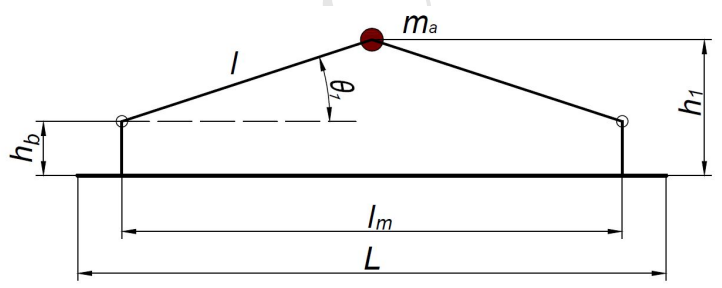

Figure 23: Mechanism geometry including control parameter $h_{1}$.

Fig. 24 shows FRF's in terms of the normalized acceleration of the beam tip in the frequency range up to $100[\mathrm{~Hz}]$ when varying angle the $\theta_{1}$. Attention is given to low-frequency range near the first natural frequency.

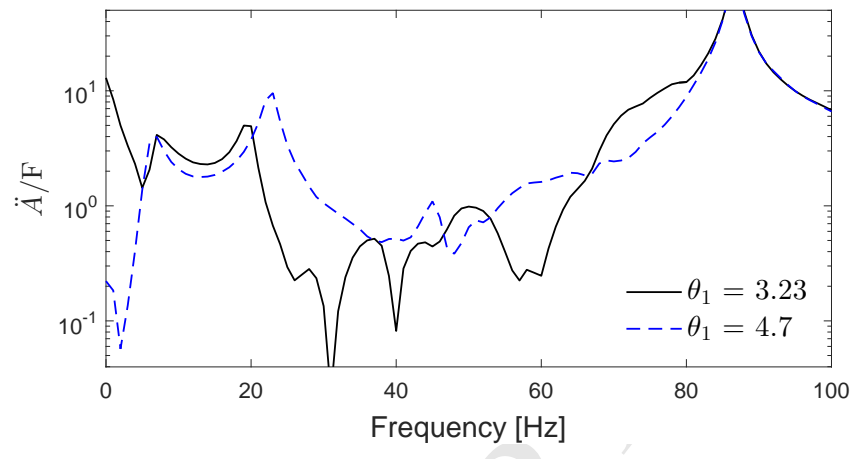

Figure 24: Angle $\theta_{1}$ variation.

It can be seen in Fig. 24 that the low-frequency attenuation properties appear to increase for decreasing angle $\theta_{1}$. A decrease of the angle to $\theta_{1}=3.23^{\circ}$ affects the first resonance peak of the system, moving it to the left, hence a wider and deeper attenuation band is observed.

Fig. 25 illustrates the effect of varying the height parameter $h_{b}$. The analysis of the distance $h_{b}$ is ambiguous due to the mentioned physical limitations of the system. To obtain representative results, the chosen FRFs correspond to system setups with $\theta_{1}$ approximately kept constant, i.e. $2.5^{\circ}$ and $2.64^{\circ}$. For those cases, it can be clearly observed that the vibration filtering properties of the mechanism increased for $h_{b}=23 \mathrm{~mm}$.

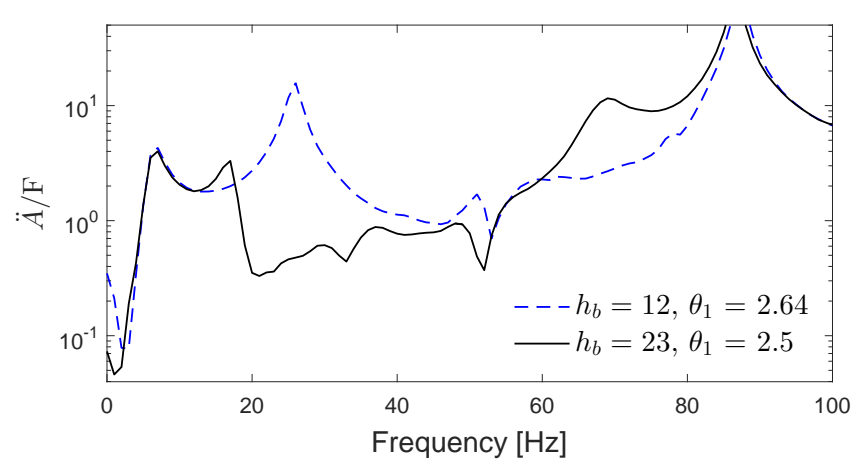

Figure 25: Bearing height $h_{b}$ variation.

The analyses of the mechanism parameters presented in Figs. 24 and 25 support the analytical predictions and numerical results presented earlier.

Fig. 26 shows the experimental FRFs when we decrease the relative length $\bar{l}$ of the mechanism as well as for the pure beam. Once more, there is a slight variation of the initial angle for the each setup, but still the desirable effect is visible. 


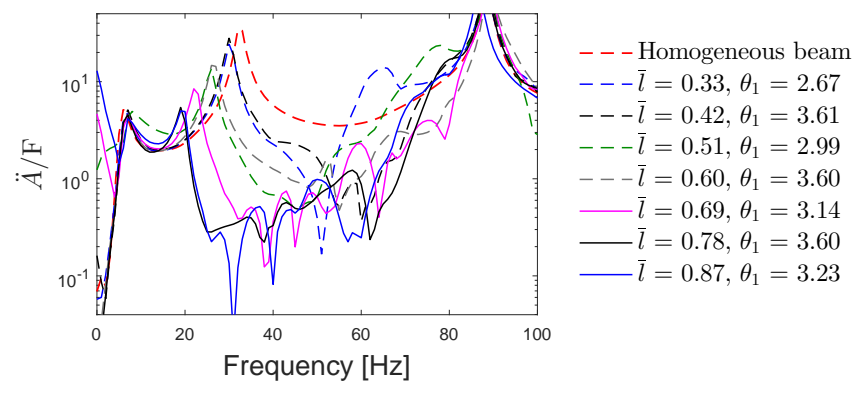

Figure 26: Effect of the mechanism width variation.

It can be seen in Fig. 26 that increasing the relative internal length increases the attenuation efficiency of the system. Furthermore, it can be observed that the FRF's approach that of the pure beam when the length of the mechanism decreases.

Many effects contribute to the observed discrepancies between experimental and numerical results. In the derived model it is assumed that the mechanism vibrates only in the $x y$-plane, and consequently, that there no motion in $z$-direction. However, for all configurations a lateral motion of the amplification mechanism was observed. The lateral vibrations are attributed to the excitation mechanism. It is very difficult to impact the beam ideally in the center and there will always be an offset leading to a twisting motion. Additionally, the rubber bands do not provide the pure parallel support as it was assumed in numerical calculations. Furthermore energy dissipation due to friction between mechanical parts is a source of discrepancy. In the numerical model this effect is not included and in order to minimize the effect, the rotational joints were thoroughly lubricated in order to decrease friction as much as it possible. This lubrication however, does not reduce the possible backlash that might occur in such bearings.

\section{Conclusion}

The attenuation of flexural vibrations in an elastic beam using an inertial amplification mechanism has been investigated by numerical and experimental means. It was demonstrated that the designed mechanism could create wide and deep gaps in the frequency response functions of the continuous beam. The gaps are generated by the inertial force that is created between two attachment points and is proportional to their relative difference in accelerations. We utilise a design similar to what is used in $[12,15]$, as a light-weight attachment to a continuous host structure similar to [15], rather than as a backbone component of a lattice structure as in [12]. This allows the mechanism to be applied directly to surfaces of structures without disturbing the functionality or integrity of the host structure.

The IA has been compared to the standard local resonator in order to benchmark its performance. For the system analysed in Fig. 12 the former generates wider and deeper gaps for the same amount of added mass.

The prominent effect of the designed system is presented in Fig. 21, where we can see the multiple anti-resonance frequencies between $1^{\text {st }}$ and $2^{\text {nd }}$ mode within the experimental FRF. This clearly illustrates the possibilities of the mechanism to generate gaps in the low-frequency range for a small amount of added mass. Fig. 21 also serves as validation of the numerical results.

In future work the attenuation of transverse vibrations in the elastic beam could be applied to more advanced structures, e.g., plates. Moreover, as it was mentioned a similar system configuration [15] was analysed with success for mechanical filtering of longitudinal vibrations. This leads to the idea of using the same mechanism for attenuating both longitudinal and transverse vibrations. Finally, the dependency on relative motion between two points also means that a similar mechanism might be used effectively as an internal component of e.g. airplane wings or wind turbine towers.

\section{Appendix A. Mechanism kinematics}

The vertical and horizontal displacements of the attachment points 1 and 2 due to the rotation of the beam cross section (see Fig. A.27) are described by:

$$
\begin{aligned}
x_{1} & =h_{b} \sin \left(\varphi_{1}\right) \\
y_{01} & =h_{b} \cos \left(\varphi_{1}\right) \\
x_{2} & =h_{b} \sin \left(\varphi_{2}\right) \\
y_{02} & =h_{b} \cos \left(\varphi_{2}\right)
\end{aligned}
$$

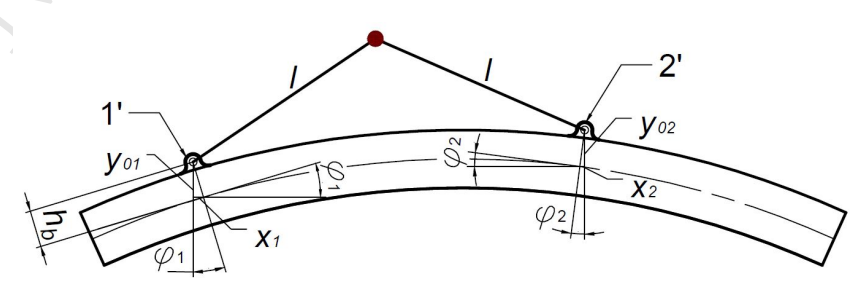

Figure A.27: The geometry after bending.

The attachment points move horizontally due to the cross section rotation. However, their total vertical displacements $y_{i i}$ are the results of an independent vertical displacements of the beam $y_{i}$ and the vertical displacements $y_{0 i}$ due to cross section rotation:

$$
\begin{aligned}
& y_{11}=y_{01}-h_{b}+y_{1} \\
& y_{22}=y_{02}-h_{b}+y_{2}
\end{aligned}
$$

As illustrated in Fig. A.29 the displacement of the additional mass in the vertical direction $y_{m}$ is expressed as a difference between its initial height $h_{1}$ and the height in the bent configuration $h_{2}$. The horizontal displacement $x_{m}$ is determined, see Eq. (A.8), by geometric relations seen in Fig. A.28 such that:

$$
\begin{aligned}
& y_{m}=h_{2}-h_{1} \\
& x_{m}=a_{21}-x_{1}-\frac{a_{1}}{2}
\end{aligned}
$$




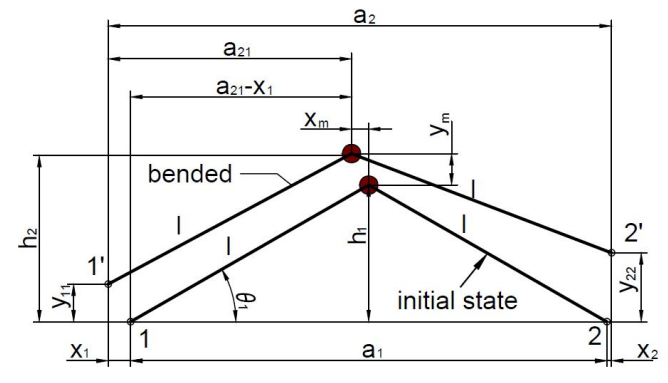

Figure A.28: Inertial mechanism in initial position and after bending.

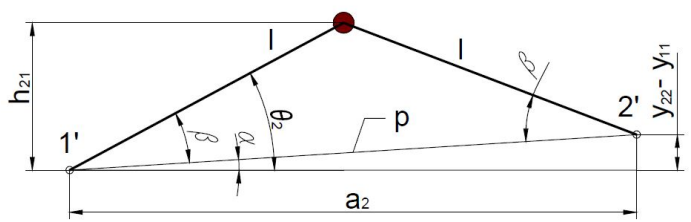

Figure A.29: Dimensions of the structure after bending.

In order to express $y_{m}$ and $x_{m}$ by the known variables, the calculation of the additional parameters based on Figs. A.28 and A.29 is required. The span of the attaching points in the initial state is:

$$
a_{1}=2 l \cos \left(\theta_{1}\right)
$$

for the bent state it becomes:

$$
a_{2}=a_{1}+x_{1}-x_{2}
$$

and the following geometric relations illustrated in Fig. A.29:

$$
\begin{aligned}
\alpha & =\arctan \left(\frac{y_{2}-y_{1}}{a_{2}}\right) \\
p & =\frac{a_{2}}{\cos (\alpha)} \\
\beta & =\arccos \left(\frac{p}{2 l}\right) \\
\theta_{2} & =\alpha+\beta \\
h_{21} & =l \sin \left(\theta_{2}\right)
\end{aligned}
$$

it leads to:

$$
\begin{aligned}
h_{2} & =h_{21}+y_{1} \\
h_{1} & =l \sin \left(\theta_{1}\right) \\
a_{21} & =l \cos \left(\theta_{2}\right)
\end{aligned}
$$

Substituting Eq. (A.9)-A.18 into Eq. (A.7), the displacement components of the mass take full form as follow:

$$
\begin{aligned}
y_{m} & =h_{b} \cos \left(\varphi_{1}\right)-h_{b}+y_{1}-l \sin \left(\theta_{1}\right) \\
& +l \sin \left(\arctan \left(\frac{h_{b} \cos \left(\varphi_{2}\right)+y_{2}-h_{b} \cos \left(\varphi_{1}\right)-y_{1}}{2 l \cos \left(\theta_{1}\right)+t \sin \left(\varphi_{1}\right)-h_{b} \sin \left(\varphi_{2}\right)}\right)\right. \\
& \left.+\arccos \left(\frac{\left.-h_{b} \sin \left(\varphi_{2}\right)\right) \sqrt{1+\frac{\left(h_{b} \cos \left(\varphi_{2}\right)+y_{2}-h_{b} \cos \left(\varphi_{1}\right)-y_{1}\right)^{2}}{\left(2 l \cos \left(\theta_{1}\right)+h_{b} \sin \left(\varphi_{1}\right)-h_{b} \sin \left(\varphi_{2}\right)\right)^{2}}}}{2 l}\right)\right)
\end{aligned}
$$

$$
\begin{aligned}
x_{m} & =-l \cos \left(\theta_{1}\right)-h_{b} \sin \left(\varphi_{1}\right) \\
& +l \cos \left(\arctan \left(\frac{h_{b} \cos \left(\varphi_{2}\right)+y_{2}-h_{b} \cos \left(\varphi_{1}\right)-y_{1}}{2 l \cos \left(\theta_{1}\right)+h_{b} \sin \left(\varphi_{1}\right)-h_{b} \sin \left(\varphi_{2}\right)}\right)\right.
\end{aligned}
$$

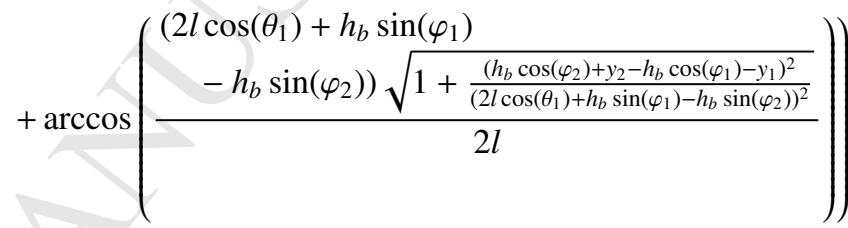

We consider linear motion, thus the equations $y_{m}$ and $x_{m}$ are linearised. It is assumed that the rotation angles $\varphi_{1}$ and $\varphi_{2}$ are relatively small so functions $\cos \left(\varphi_{i}\right)=1$ and $\sin \left(\varphi_{i}\right)=\varphi_{i}$.

$$
\begin{aligned}
& y_{m}=y_{1}-l \sin \left(\theta_{1}\right)+l \sin \left(\arctan \left(\frac{y_{2}-y_{1}}{2 l \cos \left(\theta_{1}\right)+h_{b} \varphi_{1}-h_{b} \varphi_{2}}\right)\right.
\end{aligned}
$$

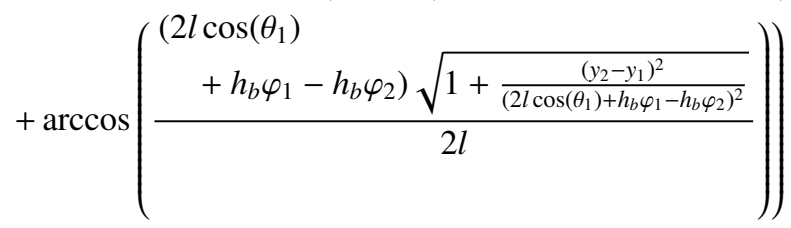

$$
\begin{aligned}
& \begin{aligned}
x_{m} & =-l \cos \left(\theta_{1}\right)-h_{b} \varphi_{1} \\
& +l \cos \left(\arctan \left(\frac{y_{2}-y_{1}}{2 l \cos \left(\theta_{1}\right)+h_{b} \varphi_{1}-h_{b} \varphi_{2}}\right)\right. \\
& \left.+\arccos \left(\frac{\left(2 l \cos \left(\theta_{1}\right)\right.}{\left.+h_{b} \varphi_{1}-h_{b} \varphi_{2}\right) \sqrt{1+\frac{\left(y_{2}-y_{1}\right)^{2}}{\left(2 l \cos \left(\theta_{1}\right)+h_{b} \varphi_{1}-h_{b} \varphi_{2}\right)^{2}}}}\right)\right)
\end{aligned}
\end{aligned}
$$

We can assume that the fractional term inside the square root vanishes for small displacements, $y_{1}$ and $y_{2}$, since $\left(y_{2}-y_{1}\right)^{2}<<\left(2 l \cos \left(\theta_{1}\right)+h_{b} \varphi_{1}-h_{b} \varphi_{2}\right)^{2}$ thus Eqs. (A.21) 
and (A.22) become:

$$
\begin{aligned}
y_{m} & =y_{1}-l \sin \left(\theta_{1}\right)+l \sin \left(\arctan \left(\frac{y_{2}-y_{1}}{2 l \cos \left(\theta_{1}\right)+h_{b} \varphi_{1}-h_{b} \varphi_{2}}\right)\right. \\
& \left.+\arccos \left(\frac{\left(2 l \cos \left(\theta_{1}\right)+h_{b} \varphi_{1}-h_{b} \varphi_{2}\right)}{2 l}\right)\right)
\end{aligned}
$$

$$
\begin{aligned}
x_{m} & =-l \cos \left(\theta_{1}\right)-h_{b} \varphi_{1}+l \cos \left(\arctan \left(\frac{y_{2}-y_{1}}{2 l \cos \left(\theta_{1}\right)+h_{b} \varphi_{1}-h_{b} \varphi_{2}}\right)\right. \\
& \left.+\arccos \left(\frac{\left(2 l \cos \left(\theta_{1}\right)+h_{b} \varphi_{1}-h_{b} \varphi_{2}\right)}{2 l}\right)\right)
\end{aligned}
$$

The trigonometric functions arctan and arccos which appear in the derivation of the displacement components are linearised by means of multiple variable Taylor series for arguments: $y_{1}$, $y_{2}, \varphi_{1}$ and $\varphi_{2}$, expanding all of them near the zero point. Thus Eqs. (A.23) and (A.24) are expressed by:

$y_{m}=y_{1}-l \sin \left(\theta_{1}\right)+l \sin \left(\frac{y_{2}-y_{1}}{2 l \cos \left(\theta_{1}\right)}+\theta_{1}-\frac{h_{b}}{2 l \sin \left(\theta_{1}\right)}\left(\varphi_{1}-\varphi_{2}\right)\right)$

$$
\begin{aligned}
x_{m} & =-l \cos \left(\theta_{1}\right)-h_{b} \varphi_{1} \\
& +l \cos \left(\frac{y_{2}-y_{1}}{2 l \cos \left(\theta_{1}\right)}+\theta_{1}-\frac{h_{b}}{2 l \sin \left(\theta_{1}\right)}\left(\varphi_{1}-\varphi_{2}\right)\right)
\end{aligned}
$$

Using once again Taylor expansion for the same arguments as above, here for the non-linear sin and cos function in Eqs. (A.25) and (A.26), finally the linearised displacement components are given as:

$$
\begin{aligned}
& y_{m}=\frac{1}{2}\left(y_{2}+y_{1}\right)-\frac{1}{2} h_{b}\left(\varphi_{1}-\varphi_{2}\right) \cot \left(\theta_{1}\right) \\
& x_{m}=\frac{1}{2}\left(-y_{2}+y_{1}\right) \tan \left(\theta_{1}\right)-\frac{1}{2} h_{b}\left(\varphi_{1}+\varphi_{2}\right)
\end{aligned}
$$

\section{Appendix B. Governing equations}

For the considered mechanism only kinetic energy exists and is given by:

$$
\begin{aligned}
T & =\frac{1}{2} m_{a}\left(y_{m}^{2}+x_{m}^{2}\right)=\frac{1}{2} m_{a}\left[\left(\frac{1}{2} \dot{y}_{2}+\frac{1}{2} \dot{y}_{1}-\frac{h_{b}\left(\dot{\varphi}_{1}-\dot{\varphi}_{2}\right)}{2 \tan \left(\theta_{1}\right)}\right)^{2}\right. \\
& \left.+\left(\frac{1}{2} \tan \left(\theta_{1}\right)\left(-\dot{y}_{2}+\dot{y}_{1}\right)-\frac{1}{2} h_{b}\left(\dot{\varphi}_{1}+\dot{\varphi}_{2}\right)\right)^{2}\right]
\end{aligned}
$$

Finding all terms of Lagrange equation:

$$
\begin{aligned}
\frac{\partial L}{\partial \dot{y}_{1}} & =\frac{1}{2} m_{a}\left[\frac{1}{2} \dot{y}_{2}+\frac{1}{2} \dot{y}_{1}-\frac{h_{b}\left(\dot{\varphi}_{1}-\dot{\varphi}_{2}\right)}{2 \tan \left(\theta_{1}\right)}\right. \\
& \left.+\left(\frac{1}{2} \tan \left(\theta_{1}\right)\left(-\dot{y}_{2}+\dot{y}_{1}\right)-\frac{1}{2} h_{b}\left(\dot{\varphi}_{1}+\dot{\varphi}_{2}\right)\right) \tan \left(\theta_{1}\right)\right] \\
\frac{\partial L}{\partial \dot{y}_{2}} & =\frac{1}{2} m_{a}\left[\frac{1}{2} \dot{y}_{2}+\frac{1}{2} \dot{y}_{1}-\frac{h_{b}\left(\dot{\varphi}_{1}-\dot{\varphi}_{2}\right)}{2 \tan \left(\theta_{1}\right)}\right. \\
& \left.-\left(\frac{1}{2} \tan \left(\theta_{1}\right)\left(-\dot{y}_{2}+\dot{y}_{1}\right)-\frac{1}{2} h_{b}\left(\dot{\varphi}_{1}+\dot{\varphi}_{2}\right)\right) \tan \left(\theta_{1}\right)\right] \\
\frac{\partial L}{\partial \dot{\varphi}_{1}} & =\frac{1}{2} m_{a}\left[-\frac{\left(\frac{1}{2} \dot{y}_{2}+\frac{1}{2} \dot{y}_{1}-\frac{h_{b}\left(\dot{\varphi}_{1}-\dot{\varphi}_{2}\right)}{2 \tan \left(\theta_{1}\right)}\right) h_{b}}{\tan \left(\theta_{1}\right)}\right. \\
& \left.-\left(\frac{1}{2} \tan \left(\theta_{1}\right)\left(-\dot{y}_{2}+\dot{y}_{1}\right)-\frac{1}{2} h_{b}\left(\dot{\varphi}_{1}+\dot{\varphi}_{2}\right)\right) h_{b}\right] \\
\frac{\partial L}{\partial \dot{\varphi}_{2}} & =\frac{1}{2} m_{a}\left[\frac{\left(\frac{1}{2} \dot{y}_{2}+\frac{1}{2} \dot{y}_{1}-\frac{h_{b}\left(\dot{\varphi}_{1}-\dot{\varphi}_{2}\right)}{2 \tan \left(\theta_{1}\right)}\right) h_{b}}{\tan \left(\theta_{1}\right)}\right. \\
& \left.-\left(\frac{1}{2} \tan \left(\theta_{1}\right)\left(-\dot{y}_{2}+\dot{y}_{1}\right)-\frac{1}{2} h_{b}\left(\dot{\varphi}_{1}+\dot{\varphi}_{2}\right)\right) h_{b}\right]
\end{aligned}
$$

then substituting them to Eq. (2), one obtains

$$
\begin{aligned}
& \frac{1}{2} m_{a}\left[\frac{1}{2} \ddot{y}_{2}+\frac{1}{2} \ddot{y}_{1}-\frac{h_{b}\left(\ddot{\varphi}_{1}-\ddot{\varphi}_{2}\right)}{2 \tan \left(\theta_{1}\right)}\right. \\
& \left.+\left(\frac{1}{2} \tan \left(\theta_{1}\right)\left(-\ddot{y}_{2}+\ddot{y}_{1}\right)-\frac{1}{2} h_{b}\left(\ddot{\varphi}_{1}+\ddot{\varphi}_{2}\right)\right) \tan \left(\theta_{1}\right)\right]=P_{1 y} \\
& \frac{1}{2} m_{a}\left[\frac{1}{2} \ddot{y}_{2}+\frac{1}{2} \ddot{y}_{1}-\frac{h_{b}\left(\ddot{\varphi}_{1}-\ddot{\varphi}_{2}\right)}{2 \tan \left(\theta_{1}\right)}\right. \\
& \left.-\left(\frac{1}{2} \tan \left(\theta_{1}\right)\left(-\ddot{y}_{2}+\ddot{y}_{1}\right)-\frac{1}{2} h_{b}\left(\ddot{\varphi}_{1}+\ddot{\varphi}_{2}\right)\right) \tan \left(\theta_{1}\right)\right]=P_{2 y} \\
& \frac{1}{2} m_{a}\left[-\frac{\left(\frac{1}{2} \ddot{y}_{2}+\frac{1}{2} \ddot{y}_{1}-\frac{h_{b}\left(\ddot{\varphi}_{1}-\ddot{\varphi}_{2}\right)}{2 \tan \left(\theta_{1}\right)}\right) h_{b}}{\tan \left(\theta_{1}\right)}\right. \\
& \left.-\left(\frac{1}{2} \tan \left(\theta_{1}\right)\left(-\ddot{y}_{2}+\ddot{y}_{1}\right)-\frac{1}{2} h_{b}\left(\dot{\varphi}_{1}+\dot{\varphi}_{2}\right)\right) h_{b}\right]=M_{1} \\
& \frac{1}{2} m_{a}\left[\frac{\left(\frac{1}{2} \ddot{y}_{2}+\frac{1}{2} \ddot{y}_{1}-\frac{h_{b}\left(\ddot{\varphi}_{1}-\ddot{\varphi}_{2}\right)}{2 \tan \left(\theta_{1}\right)}\right) h_{b}}{\tan \left(\theta_{1}\right)}\right. \\
& \left.-\left(\frac{1}{2} \tan \left(\theta_{1}\right)\left(-\ddot{y}_{2}+\ddot{y}_{1}\right)-\frac{1}{2} h_{b}\left(\ddot{\varphi}_{1}+\ddot{\varphi}_{2}\right)\right) h_{b}\right]=M_{2}
\end{aligned}
$$

and rearranging equations

$$
\begin{aligned}
& \frac{1}{4} m_{a}\left[\left(1+\tan \left(\theta_{1}\right)^{2}\right) \ddot{y}_{1}-h_{b}\left(\frac{1}{\tan \left(\theta_{1}\right)}+\tan \left(\theta_{1}\right)\right) \ddot{\varphi}_{1}\right. \\
& \left.+\left(1-\tan \left(\theta_{1}\right)^{2}\right) \ddot{y}_{2}+h_{b}\left(\frac{1}{\tan \left(\theta_{1}\right)}-\tan \left(\theta_{1}\right)\right) \ddot{\varphi}_{2}\right]=P_{1 y} \\
& \frac{1}{4} m_{a}\left[\left(1-\tan \left(\theta_{1}\right)^{2}\right) \ddot{y}_{1}-h_{b}\left(\frac{1}{\tan \left(\theta_{1}\right)}-\tan \left(\theta_{1}\right)\right) \ddot{\varphi}_{1}\right. \\
& \left.+\left(1+\tan \left(\theta_{1}\right)^{2}\right) \ddot{y}_{2}+h_{b}\left(\frac{1}{\tan \left(\theta_{1}\right)}+\tan \left(\theta_{1}\right)\right) \ddot{\varphi}_{2}\right]=P_{2 y}
\end{aligned}
$$




$$
\begin{aligned}
& \frac{1}{4} m_{a}\left[h_{b}\left(-\tan \left(\theta_{1}\right)+\frac{1}{\tan \left(\theta_{1}\right)}\right) \ddot{y}_{1}-h_{b}^{2}\left(1+\frac{1}{\tan \left(\theta_{1}\right)^{2}}\right) \ddot{\varphi}_{1}\right. \\
& \left.+h_{b}\left(\tan \left(\theta_{1}\right)-\frac{1}{\tan \left(\theta_{1}\right)}\right) \ddot{y}_{2}+h_{b}^{2}\left(1-\frac{1}{\tan \left(\theta_{1}\right)^{2}}\right) \ddot{\varphi}_{2}\right]=M_{1} \\
& \frac{1}{4} m_{a}\left[-h_{b}\left(\tan \left(\theta_{1}\right)+\frac{1}{\tan \left(\theta_{1}\right)}\right) \ddot{y}_{1}-h_{b}^{2}\left(1-\frac{1}{\tan \left(\theta_{1}\right)^{2}}\right) \ddot{\varphi}_{1}\right. \\
& \left.+h_{b}\left(\tan \left(\theta_{1}\right)+\frac{1}{\tan \left(\theta_{1}\right)}\right) \ddot{y}_{2}+h_{b}^{2}\left(1+\frac{1}{\tan \left(\theta_{1}\right)^{2}}\right) \ddot{\varphi}_{2}\right]=M_{2}
\end{aligned}
$$

leads to the governing equations of the system:

$$
\begin{aligned}
m_{1} \ddot{y}_{1}-m_{2} \ddot{\varphi}_{1}+m_{3} \ddot{y}_{2}+m_{4} \ddot{\varphi}_{2} & =P_{1 y} \\
-m_{4} \ddot{y}_{1}+m_{5} \ddot{\varphi}_{1}-m_{4} \ddot{y}_{2}+m_{6} \ddot{\varphi}_{2} & =M_{1} \\
m_{3} \ddot{y}_{1}-m_{4} \ddot{\varphi}_{1}+m_{1} \ddot{y}_{2}+m_{2} \ddot{\varphi}_{2} & =P_{2 y} \\
m_{4} \ddot{y}_{1}+m_{6} \ddot{\varphi}_{1}+m_{4} \ddot{y}_{2}+m_{5} \ddot{\varphi}_{2} & =M_{2}
\end{aligned}
$$

where the $m_{1}, m_{2}, \ldots, m_{8}$ are effective masses of the internal amplification mechanism.

\section{Appendix C. Parameters of the carbon fibre tubes}

Table C.10: Properties of the Carbon Tube

\begin{tabular}{ll}
\hline \hline Outside diameter & $10.0[\mathrm{~mm}]$ \\
Inside diameter & $8.0[\mathrm{~mm}]$ \\
Weight per m & $43.7[\mathrm{~g}]$ per meter \\
Length & $750[\mathrm{~mm}]$ \\
Structural material & $T 300$ carbon fibres \\
Matrix & Epoxy resign \\
Carbon content & Approximately $60 \%$ \\
Young modulus & $230[\mathrm{GPa}]$ \\
Ultimate tensile strength & $1600-2300[\mathrm{MPa}]$ \\
Fibre density & $1.4-1.8\left[\mathrm{~g} / \mathrm{cm}^{3}\right]$ \\
Resin glass transition Tg & $170^{\circ} \mathrm{C}$ \\
\hline \hline
\end{tabular}

\section{Appendix D. Technical data of the experimental equipment}

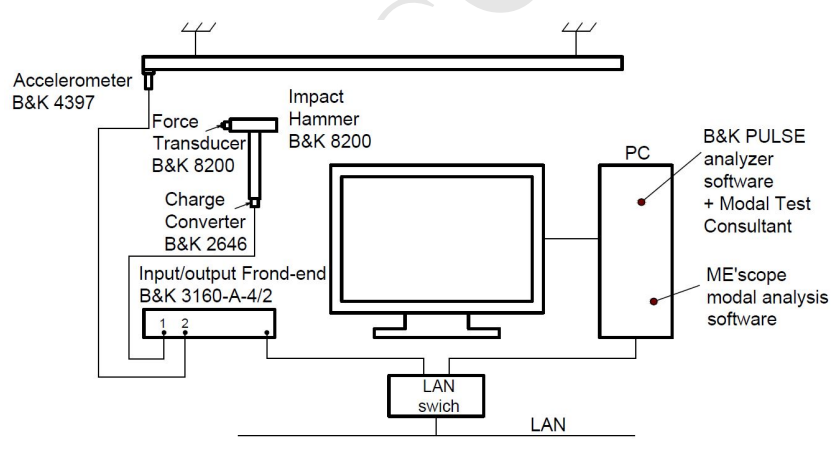

Figure D.30: Experimental setup.
Table D.11: Brüel \& Kjaer digital frequency analyser.

\begin{tabular}{lll}
\hline \hline Components & Type & Serial No. \\
\hline Modal hammer & 8206 & 57983 \\
Force transducer & 8200 & - \\
Charge converter & 2646 & - \\
Accelerometer & 4397 & 10113 \\
Generator module & $3160-A 042$ & $3160-106220$ \\
\hline \hline
\end{tabular}

\section{Acknowledgement}

This research did not receive any specific grant from funding agencies in the public, commercial, or not-for-profit sectors.

[1] H. Frahm, Device for damping vibration of bodies, U.S. Patent No. 989958, 1911.

[2] J. Ormondroyd, J. P. Den Hartog, Theory of the dynamic vibration absorber, Transactions of the ASME 50 (1928) 9-22.

[3] J. P. Den Hartog, Mechanical Vibrations, 2nd Edition, McGraw-Hill, New York, 1940.

[4] S. E. Randall, D. M. Halsted, D. L. Taylor, Optimum vibration absorbers for linear damped systems, Transactions of the ASME 103 (1981) 908913.

[5] G. B. Warburton, Optimum absorber parameters for minimizing vibration response, Earthquake Engineering and Structural Dynamics 9 (1981) $251-262$.

[6] K. C. Tsai, G. C. Lin, Optimum tuned-mass dampers for minimizing steady-state response of support-excited and damped systems, Earthquake Engineering and Structural Dynamics 22 (1993) 957-973.

[7] J. J. Connor, Structural Motion Control, Pearson Education, Inc., New Jersey, 1993.

[8] G. W. Flannelly, Dynamic antiresonant vibration isolator, U.S. Patent 989,958., 1967.

[9] M. C. Smith, Synthesis of mechanical networks: The inerter, IEEE Transactions on Automatic Control 47 (10) (2002) 1648-1662.

[10] M. Z. Q. Chen, C. Papageorgiou, F. Scheibe, F.-C. Wang, M. C. Smith, The missing mechanical circuit element, IEEE Circuits and Systems Magazine 9 (2009) 10-26.

[11] C. Yilmaz, N. Kikuchi, Analysis and design of passive low-pass filter-type vibration isolators considering stiffness and mass limitations, Journal of Sound and Vibration 293 (2006) 171-195.

[12] C. Yilmaz, G. M. Hulbert, N. Kikuchi, Phononic band gaps induced by inertial amplification in periodic media, Physical Review B 76 (2007) 054309.

[13] C. Yilmaz, G. M. Hulbert, Theory of phononic gaps induced by inertial amplification in finite structures, Physics Letters A 374 (2010) 35763584.

[14] G. Acar, C. Yilmaz, Experimental and numerical evidence for the existence of wide and deep phononic gaps induced by inertial amplification in two-dimensional solid structures, Journal of Sound and Vibration 332 (2013) 6389-6404.

[15] N. M. M. Frandsen, O. R. Bilal, J. S. Jensen, M. I. Hussein, Inertial amplification of continuous structures: Large band gaps from small masses, Journal of Applied Physics 119 (2016) 124902.

[16] J. J. Thomsen, Vibrations and Stability. Advanced Theory, Analysis, and Tools, 2nd Edition, Springer, 2003.

[17] S. Krenk, J. Høgsberg, Tuned resonant mass or inerter-based absorbers: unified calibration with quasi-dynamic flexibility and inertia correction, Proc. R. Soc. A 472 (2016) 20150718.

[18] K. L. Lawrence, ANSYS ${ }^{\circledR}$ Tutorial. Structural \& Thermal Analysis Using the ANSYS Mechanical APDL. Release 13 Environment, Schorff Development Corporation, 2011.

[19] Brüel, Kjaer, PULSE - Getting Started. An Introduction to PULSE, Brüel \& Kjaer Sound \& Vibration Measurement A/S, Naerum, Denmark (9 2003). 


\section{Highlights:}

- An inertial amplification mechanism for attenuation of beam vibrations is analyzed.

- The mechanism generates multiple anti-resonance dips in the low-frequency range.

- Improved efficiency in comparison to a classical local resonance system.

- The obtained numerical results are validated with the experimental data. 